\title{
Fast summation of double infinite modal series using the Poisson summation formula
}

\author{
Piotr M. Słobodzian \\ Institute of Telecommunications and Acoustics, Wroctaw University of Technology \\ Wyb. Wyspiańskiego 27, 50-370 Wrocław, Poland \\ e-mail: Piotr.Slobodzian@pwr.wroc.pl
}

(Rec. 17 June 2004)

\begin{abstract}
The main aim of this work is to verify the effectiveness of Poisson's transformation in the summation of multiple-valued, double infinite modal series, encountered in the computational electromagnetism related to analysis of shielded microstrip circuits. In this contribution, the Poisson summation formula has been applied to accelerate the rate of convergence of the static part of the modal series under consideration in order to enable the effective application of Kummer's transformation. The need for the use of Poisson's formula has resulted from the fact that the studied modal series is a multiple-valued one and hence the conventional approach based on the complex contour integral method can not be exploited. Finally, the use of Kummer's transformation in conjunction with Poisson's summation formula has proved to be very efficient and enabled radical savings in computational time. This feature makes the proposed method a good candidate for practical applications, especially for electromagnetic CAD tools.
\end{abstract}

Key words: series convergence acceleration, series transformation, Poisson's summation formula, IE-MoM approach

\section{INTRODUCTION}

The double infinite modal series arise in the full-wave analysis of shielded microstrip circuits by means of the integral equations - method of moments (IE-MoM) approach. A method for its efficient summation, based on Kummer's transformation and the complex contour integral method, has been recently described in [1]. Unfortunately, the proposed method can not be directly applied to multiple-valued series with summand having branch points, which are not isolated singularities. In such a case, the residue theorem can not be exploited and the complex contour integral, involved in the series transformation, includes an integration along branch cuts, which must be evaluated numerically. This is, obviously, a task that entails a huge computational effort what makes the approach highly ineffective. Nevertheless, it is still possible to accelerate the rate of convergence of such the modal series by using the Poisson summation formula. It has been shown that this integral transformation proved to be very effective in the accelerated summation of periodic, free-space Green's functions, which take the form of multiple-valued series [2]. Hence, the main aim of this contribution is to verify the usefulness of Poisson's transformation in the summation of double infinite modal series arising during analysis of vertical current sources embedded in a shielded multilayered medium.

The method for series transformation, described in this paper, is adopted partly form [1] and consist of two stages.
Firstly, the modal series under consideration will be transformed by means of Kummer's method, and than the resulting double infinite series will be transformed into single infinite ones. Secondly, we shall discuss the possibility of the Poisson summation formula application. In order to demonstrate the proposed method for series transformation we shall apply it to one of the modal series, which has been already described in [1], namely the series $Z_{3}$, which is a multiple-valued series.

\section{SERIES TRANSFORMATION}

The double infinite modal series under consideration is associated with a diagonal entry of the MoM matrix resulting from the analysis of a vertical strip embedded in a rectangular cavity filled with an isotropic, linear medium [1]. The series has the following form

$$
\begin{aligned}
& Z_{3}=-4 \cdot \sum_{m=1}^{\infty} \sum_{n=1}^{\infty} \frac{W_{m n}}{k_{y_{n}}^{2}} \cdot \frac{k_{\rho_{m n}}^{2}}{\beta_{m n}^{5}}= \\
& =-4 \cdot \sum_{m=1}^{\infty} \sum_{n=1}^{\infty} \frac{W_{m n}}{k_{y_{n}}^{2}} \cdot \frac{k_{\rho_{m n}}^{2}}{\left(\sqrt{k_{o}^{2}-k_{\rho_{m n}}^{2}}\right)^{5}},
\end{aligned}
$$

where

$$
W_{m n}=\sin ^{2}\left(k_{x_{m}} x_{o}\right) \cdot\left[\cos \left(k_{y_{n}} y_{1}\right)-\cos \left(k_{y_{n}} y_{2}\right)\right]^{2},
$$


and

$$
k_{\rho_{m n}}=\sqrt{k_{x_{m}}^{2}+k_{y_{n}}^{2}}, \quad k_{x_{m}}=\frac{m \pi}{a}
$$

$$
k_{y_{n}}=\frac{n \pi}{b}
$$

and where, all the remaining quantities have been defined and described in [1]. As we can see, the series in Eq. (1) is a double-valued series with complex terms due to the square root standing in the denominator of the summand. This property directly impinges on the possibility of application of the integral representation of the series, but this issue will be discussed somewhat later. Now, we shall focus on the first stage of the series transformation, i.e. we shall transform the series $Z_{3}$ via Kummer's transformation.

The concept of the Kummer's transformation has been described in [1]. Upon applying this transformation to the series in Eq. (1), provided that its static part is determined for $f \rightarrow 0$, we can write

$$
Z_{3}=Z_{3}^{\text {stat }}+Z_{3}^{\text {dyn }}
$$

where

$$
\begin{gathered}
Z_{3}^{\text {stat }}=-4 j \cdot \sum_{m=1}^{\infty} \sum_{n=1}^{\infty} \frac{W_{m n}}{k_{y_{n}}^{2}} \cdot \frac{1}{k_{\rho_{m n}}^{3}}, \\
Z_{3}^{\text {dyn }}=-4 \cdot \sum_{m=1}^{\infty} \sum_{n=1}^{\infty} \frac{W_{m n}}{k_{y_{n}}^{2}} \cdot\left(\frac{k_{\rho_{m n}}^{5}-j \beta_{m n}^{5}}{k_{\rho_{m n}}^{3} \cdot \beta_{m n}^{5}}\right) .
\end{gathered}
$$

The gain resulting from the use of Kummer's transformation pertains to a reduction in computational effort due to the fact that $Z_{3}^{\text {stat }}$ does not depend on the frequency at which circuit analysis is carried out. Hence, the computations in the frequency loop confine to evaluation of $Z_{3}^{\mathrm{dyn}}$, which is a rapidly converging series, and then to addition of the obtained result to the previously evaluated static part $Z_{3}^{\text {stat }}$. Although the static part is evaluated only once (before the frequency loop is entered) the involved computational effort is very high and is almost equal to that associated with the original series. Therefore, we shall focus now on the integral transformation of $Z_{3}^{\text {stat }}$ that will enable its fast summation.

To proceed, we shall adopt the method described in [1] and try to transform the double infinite series $Z_{3}^{\text {stat }}$ into a single infinite one using an integral transformation. Consequently, we shall rewrite $Z_{3}^{\text {stat }}$ in the following form

$$
Z_{3}^{\text {stat }}=-4 j \cdot \sum_{m=1}^{\infty} \sum_{n=1}^{\infty} F_{n} \cdot G_{m n}=-4 j \cdot \sum_{n=1}^{\infty}\left(F_{n} \cdot G_{n}\right),
$$

where

$$
\begin{aligned}
F_{n} & =\frac{\left[\cos \left(k_{y_{n}} y_{1}\right)-\cos \left(k_{y_{n}} y_{2}\right)\right]^{2}}{k_{y_{n}}^{2}}, \\
G_{n} & =\sum_{m=1}^{\infty} G_{m n}=\sum_{m=1}^{\infty} \frac{\sin ^{2}\left(k_{x_{m}} x_{o}\right)}{k_{\rho_{m n}}^{3}}= \\
& =\left(\frac{a}{\pi}\right)^{3} \cdot \sum_{m=1}^{\infty} \frac{\sin ^{2}\left(m \frac{\alpha}{2}\right)}{\left(m^{2}+\beta_{n}^{2}\right)^{3 / 2}}
\end{aligned}
$$

and where

$$
\begin{gathered}
\beta_{n}=n \cdot \frac{a}{b}, \\
\alpha=2 \pi \cdot \frac{x_{o}}{a} .
\end{gathered}
$$

Before we proceed with the integral transformation, let us simplify the form of $G_{n}$ by rewriting it as

$$
G_{n}=\frac{1}{4}\left(\frac{a}{\pi}\right)^{3} \cdot\left(2 \cdot S_{1}+S_{2}-\frac{1}{\beta_{n}^{3}}\right),
$$

where

$$
S_{1}=\sum_{m=1}^{\infty} \frac{1}{\left(m^{2}+\beta_{n}^{2}\right)^{3 / 2}}
$$

$$
S_{2}=\sum_{m=-\infty}^{\infty} \frac{e^{j \cdot m \alpha}}{\left(m^{2}+\beta_{n}^{2}\right)^{3 / 2}} .
$$

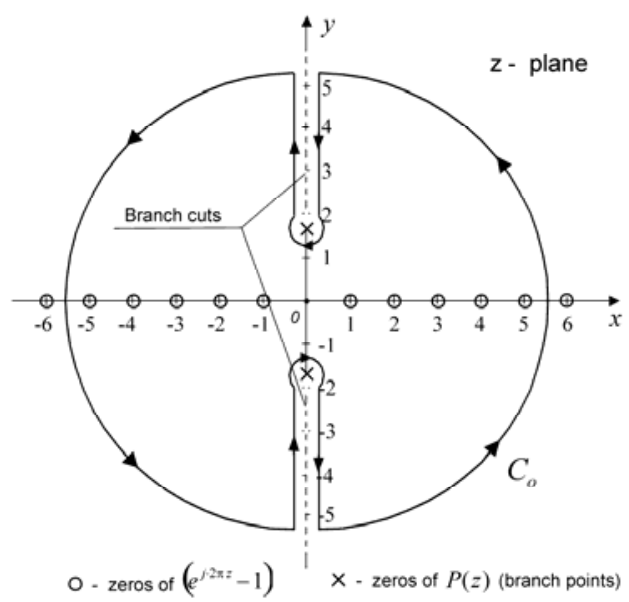

Fig 1. The contour of integration assumed for the integral representation of the multiple-valued series $S_{2}$ 
With these equations, our further considerations can be restricted to the treatment of the series $S_{2}$ and its asymptotic form for $\alpha \rightarrow 0$, which, finally, gives the series $S_{1}$.

Following the method described in [1], the series $S_{2}$ can be expressed by means of the complex contour integral of the following function

$$
F(z)=\frac{e^{j \cdot \alpha z}}{P(z)} \cdot \frac{1}{e^{j \cdot 2 \pi z}-1},
$$

where $P(z)=\left(z^{2}+\beta_{n}^{2}\right)^{3 / 2}$, with the path of integration shown in Fig. 1. Since $F(z)$ is a multiple-valued function of $z$, the $z$-plane is cut into two Riemann's sheets by two branch cuts, which have been defined in the way that the integral representation of $S_{2}$ is enabled. Additionally, the path of integration must lie in the proper Riemann sheet in order to assure physical meaning of the integral. As a consequence, the contour $C_{o}$ must avoid the both branch cuts, as shown in Fig. 1, and this gives rise to a non-zero contribution to the closed-loop integral of $F(z)$, which can be mainly attributed to the integration along the branch cuts and around the branch points. Unfortunately, in our case the branch points are non-isolated singularities and hence the residue theorem can not be used to facilitate the integral evaluation. In practice the contribution, especially related to the branch points, must be evaluated numerically and this makes the approach highly ineffective. This problem can be solved, as it has been already mentioned in the introduction, by means of the Poisson transformation, which has proved to be a good solution for a certain type of multiplevalued series, often encountered in the computational electromagnetism (see, e.g. [2]). The question of the use of Poisson's summation formula to accelerate the summation of the series $S_{2}$ will be addressed in the next section.

\section{THE POISSON TRANSFORMATION}

The Poisson transformation is based on Poisson's summation formula [3], which allows us to transform a slowly converging series into a rapidly converging one. In certain cases, this transformation allows the sum of a series to be evaluated in the closed form and such a case will be of great importance for our further considerations.

When we take into consideration a single infinite series the Poisson summation formula takes the following form

$$
\sum_{m=-\infty}^{\infty} f(\alpha \cdot m)=\frac{1}{\alpha} \cdot \sum_{m=-\infty}^{\infty} F\left(\frac{2 \pi \cdot m}{\alpha}\right),
$$

where $\quad F(u)=\mathscr{F}\{f(x)\}=\int_{-\infty}^{\infty} f(x) \cdot e^{j \cdot x u} d x$,

and where $\mathfrak{F}\{f(x)\}$ denotes the Fourier transform of $f(x)$. Consequently, in the case of the series $S_{2}$ we need to determine the Fourier transform of the summand upon evaluation of the following integral

$$
\begin{gathered}
F(u)=\int_{-\infty}^{\infty} \frac{\alpha^{3} \cdot e^{j \cdot v(u+1)}}{\left(v^{2}+\beta_{o_{n}}^{2}\right)^{3 / 2}} d v= \\
=2 \alpha^{3} \cdot \int_{0}^{\infty} \frac{\cos [v(u+1)]}{\left(v^{2}+\beta_{o_{n}}^{2}\right)^{3 / 2}} d v,
\end{gathered}
$$

where $v=\alpha \cdot m$ and $\beta_{o_{n}}=\alpha \cdot \beta_{n}$. The integral in Eq. (15) can not be evaluated in the closed form and expressed by means of the elementary transcendental functions. On the other hand, the integral constitutes an integral representation of the modified Bessel function $K_{v}(z)$ of the integer order $v$, which for $v=1$ has the following form (see, Eq. (9.6.25), p. 376 in [4])

$$
K_{1}(z \cdot u)=\frac{2 u \cdot \Gamma\left(\frac{3}{2}\right)}{z \cdot \sqrt{\pi}} \cdot \int_{0}^{\infty} \frac{\cos (v \cdot z)}{\left(v^{2}+u^{2}\right)^{3 / 2}} d v
$$

where $\Gamma$ stands for the Gamma function and $z \in R>0$, and $\arg (u)<\pi / 2$. Upon substituting Eq. (16) into Eq. (15) and then using Eq. (13) we can, finally, express the sum of the series $S_{2}$ as

$$
\begin{gathered}
S_{2}=\sum_{m=-\infty}^{\infty} \frac{e^{j \cdot m \alpha}}{\left(m^{2}+\beta_{n}^{2}\right)^{3 / 2}}= \\
=\frac{2}{\beta_{n}} \cdot \sum_{m=-\infty}^{\infty} \sqrt{(2 \pi \cdot m+\alpha)^{2}} \cdot K_{1}\left(\beta_{n} \cdot \sqrt{(2 \pi \cdot m+\alpha)^{2}}\right)
\end{gathered}
$$

As we can see, the use of Poisson's summation formula has allowed the original infinite series to be transformed into another infinite series. Taking this fact into account we can conclude, at first sight, that the gain resulting from such transformation is very slight, since the new form of $S_{2}$ can possibly require even higher computational effort due to evaluation of the Bessel function $K_{1}$. However, before drawing the final conclusion concerning the rate of convergence of the new series we need to discus its properties.

When it comes to practical applications, namely the electromagnetic analysis of a vertical strip embedded in a rectangular cavity [1], all arguments of the Bessel function in Eq. (17) are real (see, the definition in Eq. (9)). Hence, $K_{1}$ is real, positive and strictly decreasing, with a very high rate of decrease (see, [4] for details). Therefore the most significant contribution to the final sum of $S_{2}$ comes mainly from such terms of the series for which the argument of $K_{1}$ is very small. In our case, such terms are related to all the indexes $m$ for which $\left(\sqrt{(2 \pi \cdot m+\alpha)^{2}} \rightarrow 0\right)$, i.e. $m \in\left\{\ldots, m_{o}-1, m_{o}, m_{o}+1, \ldots\right\}$, where

$$
m_{o}=\left[-\frac{\alpha}{2 \pi}\right]=\left[-\frac{x_{o}}{a}\right]
$$


Since, in practice $0<x_{o} / a<1$, we always have $m_{o} \in\{-1,0\}$, and in addition to this, $K_{1}$ decays very rapidly as $|m|$ increases, hence, the sum of the series $S_{2}$ can be evaluated upon summation of only several adjacent terms around $m_{o}$.

In view of the foregoing facts, the Poisson summation formula should prove very effective in the summation of the double infinite modal series under consideration. All details concerning validation of this viewpoint will be addressed in the next section.

\section{NUMERICAL EXAMPLE}

In order to verify usefulness of the Poisson transformation in the modal series summation we shall analyze the relative error of the series $Z_{3}$ sum. All calculations, described in this section, have been carried out under the following assumptions: $a \times b \times c=30 \times 20 \times 10 \mathrm{~mm}, x_{o}=10 \mathrm{~mm}$, $y_{1}=4 \mathrm{~mm}, y_{2}=6 \mathrm{~mm}, \Delta_{z}=2.2 \mathrm{~mm}, f=5.5 \mathrm{GHz}$ and $k_{o}=2 \pi f \sqrt{\mu_{0} \varepsilon_{0}}$. However, before we proceed we shall verify the effectiveness of Poisson's summation formula in the evaluation of the sum of the single infinite series $S_{2}$.

As we can see from Eq. (17), the sum of $S_{2}$ depends on the value of $\beta_{n}$, which, on the other hand, depends on the index $n$. This index strongly affects the rate of convergence of the both form of $S_{2}$ (the original and the transformed ones). Consequently, when the direct summation method is applied to the evaluation of $S_{2}=\Sigma_{m=-M}^{M} \cdots$, the number of terms $M$, which must be taken into account in order to obtain the required level of the relative error of the series sum, increases very rapidly as $n$ is on the increase (see, Fig. 2). Therefore, when evaluating the sum of the double series $Z_{3}$, in practice, a huge number of terms along the index $m$ must be summed up to assure an accurate and stable result. This is not the case when the transformed form of $S_{2}$ is used and it suffices to apply a single term approximation (i.e. for $m=m_{o}$ ) in order to get a very low level of the relative error of the series sum (see, Fig. 3), especially for sufficiently large values of $n$ (i.e. usually for $n>3$ ). In addition to this, it has been noted that for most of practical cases it suffices to use only the three-term approximation when $n \leq 3$ (i.e. for $\left.m=m_{o}-1, m_{o}, m_{o}+1\right)$. It is also worth to note that the results of calculation, shown in Fig. 2 and 3, have been obtained for the exact value of the sum $S_{2}$ evaluated using the transformed form of $S_{2}$ with $M=10$. In summary, the Poisson transformation has allowed the rate of convergence of the series $S_{2}$ to be extremely increased, so finally, it suffices to sum up at most several single terms to obtain the required accuracy of the series sum.

Having determined the usefulness of Poisson's summation formula in the transformation of the series $S_{2}$, we are in a position to analyze the results of calculation obtained for the double infinite series $Z_{3}$. Using Eq. (17) and the three-term approximation of $S_{2}$, we can transform
$Z_{3}^{\text {stat }}$, by means of Eq. (6), into the single infinite series that depends only on the index $n$. The results of calculation, shown in Fig. 4, reveal that the transformation greatly enhance the rate of convergence of $Z_{3}^{\text {stat }}$, and it suffices to take less then 100 terms to obtain very accurate results.

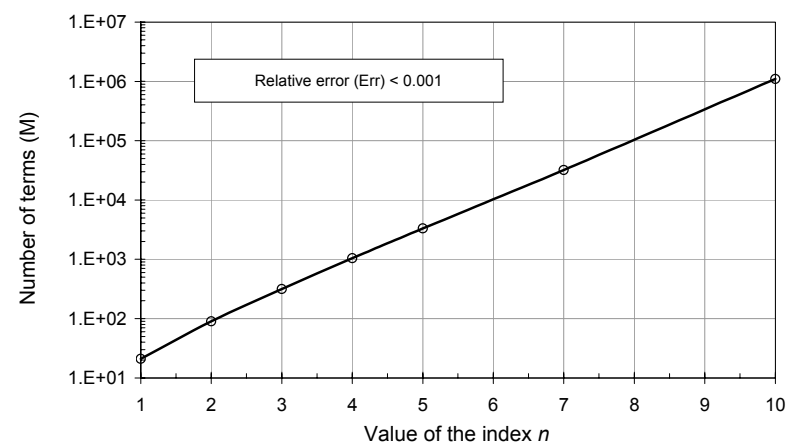

Fig 2. Number of terms required in the direct summation of the series $S_{2}$ in order to obtain the relative error of the series sum below $10^{-3}$ versus the value of the index $n$

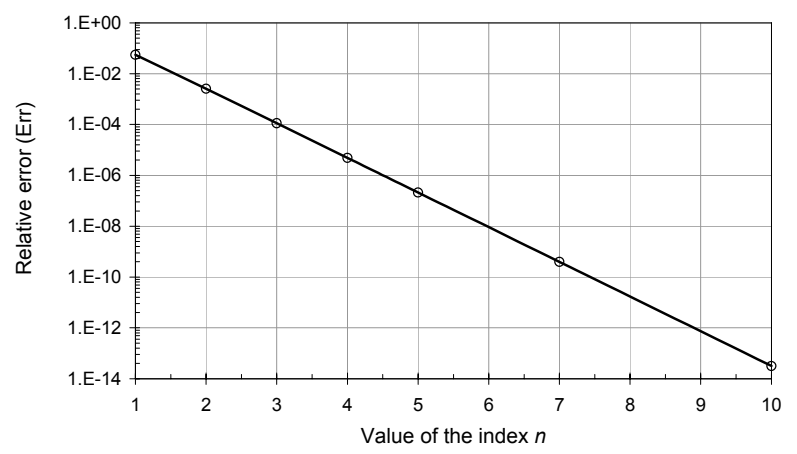

Fig 3. The relative error of the series $S_{2}$ sum versus the value of the index $n$, calculated using the single-term approximation of $S_{2}$ resulting from the Poisson summation formula

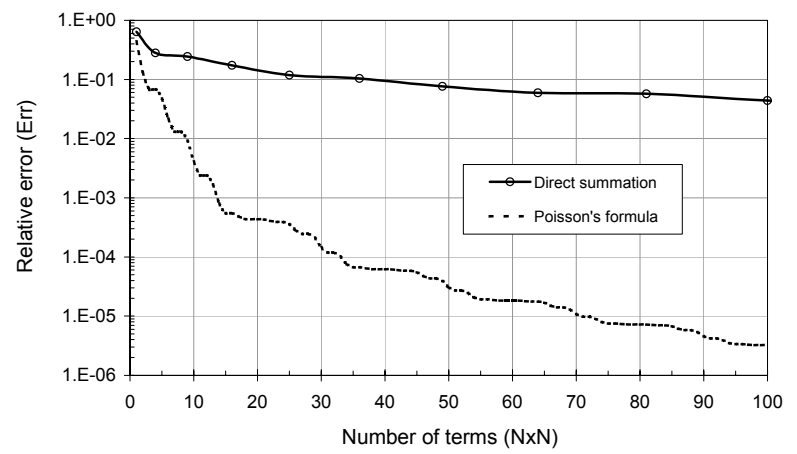

Fig 4. The rate of convergence of the static part of the series $Z_{3}$, obtained for the direct summation method and the Poisson summation formula (the direct summation method uses the square set of terms, i.e. containing $(M=N) \times N$ terms $)$ 


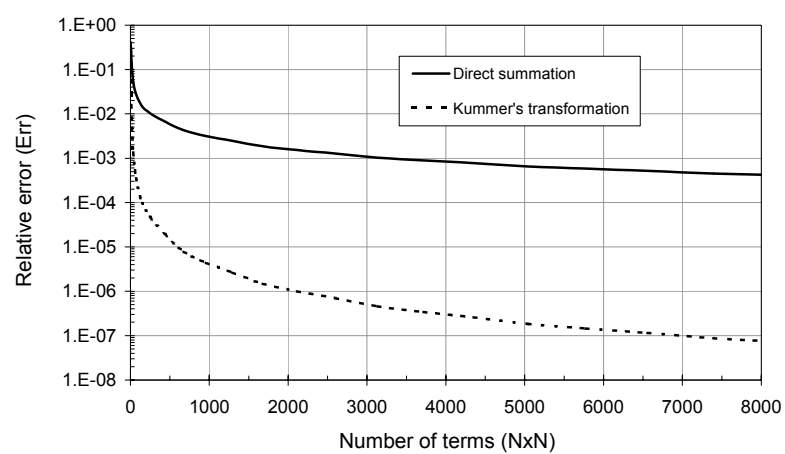

Fig 5. The rate of convergence of the series $Z_{3}$, obtained for the direct summation method and the Kummer transformation

Once the static part of the series $Z_{3}$ has been determined we can exploit Kummer's transformation, described in Eq. (3), to calculate very effectively the sum of $Z_{3}$ in the frequency loop. This time, the calculations can be restricted to the direct evaluation of the dynamic part of $Z_{3}$ (defined in Eq. (5)) since in practice $Z_{3}^{\text {dyn }}$ converges very rapidly. Consequently, the use of Kummer's transformation in conjunction with the Poisson summation formula allows the sum of the original series $Z_{3}$ to be evaluated very effectively, and the final results of calculation are shown in Fig. 5.

\section{CONCLUSIONS}

The paper describes the method for very fast evaluation of the multiple-valued, double infinite modal series, which arise in the analysis of shielded microstrip circuits in the frame of the IE-MoM approach. It has been shown that in such a case the application of the double-to-single series transformation, which allows us to evaluate the sum of the modal series very effectively, can not be accomplished by means of the complex contour integral and the residuum theorem. This problem can be solved by means of Poisson's summation formula, which has been exploited to transform the static part of the modal series into the very rapidly converging single infinite series. Finally, it has been shown that the use of Poisson's formula has enabled very effective application of the Kummer transformation, which significantly reduces the computational effort required to evaluate the sum of the modal series under consideration.

In summary, the proposed method proved to work very effectively, but its use requires much analytical work, which must be done in order to derive the Fourier transform involved in the Poisson summation formula.

\section{References}

[1] P. M. Słobodzian, Fast summation of double infinite modal series involved in analysis of shielded microstrip circuits, CMST 10, 5-15 (2003).

[2] R. Lampe, P. Klock and P. Mayes, Integral Transforms Useful for the Accelerated Summation of Periodic, FreeSpace Green's Functions, IEEE Trans. Microwave Theory and Tech., 33, 734-736 (1985).

[3] P. M. Morse and H. Feshbach, Methods of theoretical physics, McGraw-Hill Book Co., Inc., New York, 1953.

[4] M. Abramowitz and I. A. Stegun, Handbook of mathematical functions, Dover Pub., Inc., New York, 1970.

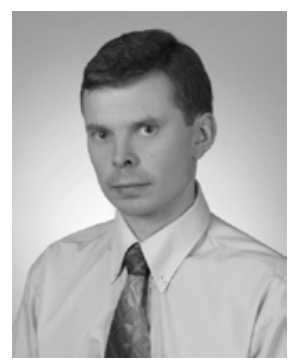

Piotr M. SŁobodzian was born in Gryfów Śląski, Poland, in 1968. He received the M.Sc. and Ph.D. degrees from the Wrocław University of Technology, Wrocław, Poland, in 1993 and 1998, respectively. Since 1998, he has been with the Radio Department, Institute of Telecommunications and Acoustics, Wrocław University of Technology, where he is currently an Assistant Professor. In 1999, he received the Swiss Fellowship and joined for nine months the Laboratory of Electromagnetics and Acoustics at the Swiss Federal Institute of Technology (LEMA-EPFL), Lausanne, Switzerland, where he started his work concerning application of the Integral Equations - Method of Moments (IE-MoM) approach in analysis of shielded microstrip circuits. His research interests focus on computational electromagnetics, microstrip antenna theory and technology, and antenna measurement techniques. 